\title{
Mid-Infrared Surface-Emitting Two Dimensional Photonic Crystal Semiconductor Lasers
}

\author{
Binbin Weng and Zhisheng Shi \\ School of Electrical and Computer Engineering \\ University of Oklahoma, Norman Oklahoma
}

USA

\section{Introduction}

The mid-infrared (mid-IR) region, covering the electromagnetic spectrum from about 2.5 to $25 \mu \mathrm{m}$ wavelength, is of unique interest for many applications, especially for molecular spectroscopy, because the vibrational frequencies of almost all target molecules and hydrocarbons are in this spectral region, such as $\mathrm{NO}, \mathrm{NO}_{2}, \mathrm{CO}, \mathrm{CO}_{2}, \mathrm{HF}$, and $\mathrm{CH}_{4}$. The specific application areas for molecular spectroscopy include scientific research, vehicle exhaust investigation, atmospheric pollution monitoring, medical diagnostics and biological \& chemical weapon detection. Besides molecular spectroscopy applications, there are also some other potential applications, such as free space optical communications, etc. (Tittel, Richter, \& Fried, 2003)

For all these applications, typical requirements for mid-IR lasers are manifold. First of all, to reach high spectral selectivity performance, a narrow spectral linewidth is needed. Secondly, optical output power should be larger than $100 \mu \mathrm{W}$ to lift detector noise limits. (Tacke M. , 2001) Thirdly, high beam quality is favorable for optimum coupling with the gas sampling cell. Also, room temperature or thermoelectric-cooled operation is desirable, considering the additional weight, volumes and costs with the additional cryogenic cooling equipment. Furthermore, a compact set-up is preferable for hand-held in situ measurement in the field.

Two dimensional (2D) photonic crystal lasers, potentially have great practical applications in the mid-IR region due to their unique features in, for example, surface emission, circular beams, low threshold operation, miniaturization and simplicity of on-chip monolithic integration, etc. The studies of surface emitting 2D photonic crystal lasers started in and have expanded worldwide since the middle of the 1990s. The first 2D photonic crystal semiconductor laser of $1.55 \mu \mathrm{m}$ emission peak was demonstrated in 1999. (Painter, et al., 1999) After that, this type of semiconductor lasers has been developed rapidly in near IR region. (Park, Hwang, Huh, Ryu, \& Lee, 2001; Loncar, Yoshie, Scherer, Gogna, \& Qiu, 2002; Altug, Englund, \& Vuckovic, 2006) Unfortunately, mainly restricted by the etch-induced surface recombination, the research development of 2D photonic crystal semiconductor lasers in mid-IR range of electromagnetic spectrum was relatively slow. Even so, due to their intrinsic advantages addressed previously, this type of lasers operating in mid-IR range is 
still very desirable. Therefore, some major steps of the progress are very important and need to be paid close attention nowadays.

In this chapter, we will review the development of the mid-IR surface emitting 2D photonic crystal semiconductor lasers in recent decade. The performance of the reported mid-IR 2D photonic crystal lasers with different structure design and different material system is going to be introduced and compared. Their major advantages and disadvantages will also be discussed.

\section{Mid-IR surface emitting 2D photonic crystal lasers}

The researches on the mid-IR semiconductor sources and structures for optoelectronics devices applications have been developed for several decades. Recently, the most competitive mid-IR semiconductor lasers are realized with GaAs- and GaSb-based IIIV material system making use of their unique intersubband (Bauer, et al., 2011; Yu, Darvish, Evans, Nguyen, Slivken, \& Razeghi, 2006; Slivken, Evans, Zhang, \& Razeghi, 2007) and inter-band (Yang R. Q., 1995; Yang, Bradshaw, Bruno, Pham, Wortman, \& Tober, 2002) cascade transition mechanisms. Meanwhile, thanks to their suppressed Auger non-radiative loss, (Zhao, Wu, Majumdar, \& Shi, 2003) IV-VI lead-salt materials have also been an excellent choice of mid-IR lasers for gas sensing application, and will continue to be so in the future. (Springholz, Schwarzl, \& Heiss, 2006) In the following, we will introduce the development of mid-IR surface emitting photonic crystal lasers using all mentioned intersubband cascade, type-II "W" active region III-V semiconductors, and leadsalt materials.

\subsection{Quantum cascade surface-emitting photonic crystal laser}

Quantum cascade laser (QCL) is a type of mid-IR laser based on intersubband transitions in multiple quantum wells for photon generation as shown in figure 1. As a result, the quantum efficiencies would be greater than $100 \%$. On the other hand, the emission wavelength of QCL is able to be tuned from 4 to $24 \mu \mathrm{m}$ in the mid-IR range. (Yu, Darvish, Evans, Nguyen, Slivken, \& Razeghi, 2006; Diehl, et al., 2006) However, most of the QCL works have been done were focused on edge-emitting lasers, and limited by its intrinsic transverse-magnetic polarization of the intersubband transition.

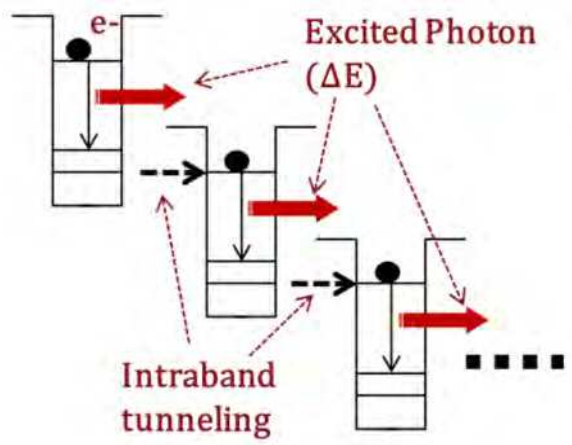

Fig. 1. The schematic of physics mechanism for quantum cascade laser (QCL) 
Fortunately, 2D photonic crystal technology allows quantum cascade lasers to achieve surface emission. The first mid-IR surface emitting 2D photonic crystal QCL was realized in 2003. (Colombelli F. , et al., 2003) In this demonstration, the 2D arrays were fabricated with the values of lattice spacing $a$ from 2.69 to $3.00 \mu \mathrm{m}$, and the ratio of holes radius $r$ to $a$ from 0.28 to 0.32 . As presented in figure $2 \mathrm{~B}$ and figure $2 \mathrm{C}$, the etched photonic crystal pattern penetrated through the active region down into the bottom substrate, which reduces the diffraction of radiation into the substrate and consequently provides strong in-plane optical feedback. Usually, in diode lasers, deep-etching induced surface recombination would substantially increase the leakage currents. (Colombelli F. , et al., 2003) But this serious obstacle does not affect in this case, because QCL are unipolar devices.

After processing, electroluminescence (EL) measurements were conducted at a temperature of $10 \mathrm{~K}$ and the light spectra were acquired using a Nicolet Fourier transform infrared (FTIR) spectrometer and a nitrogen-cooled $\mathrm{HgCdTe}$ detector. From the measurement, for the designed structure with a set of values of a and $r$ having the $A, B$, and $C$ flat-band regions overlapping the gain spectrum, three line-narrowing emission peaks emerge from the EL spectrum, as shown in Figure 3A.

It is worth to mention that, no defect mode emission has been demonstrated, because of the little difference observed between the emission spectra with and without a central defect design. (Colombelli F. , et al., 2003) Therefore, the device should be classified as the bandedge mode photonic crystal lasers. Simply explaining, the optical gain is intensified by reducing the group velocity of light, supported by a basic relation about light amplification in which (H.C. Casey \& Panish, 1978)

$$
\gamma=g N v_{g}
$$

where $\gamma$ is the net stimulated-emission rate, $g$ is the optical gain, $\mathrm{N}$ is the photon density, and $v_{g}$ is the group velocity of light. It has been proven that the gain enhancement is strongly correlated with the group velocity, which decreases rapidly in the vicinity of the photonic band edges. (Nojima, 1998)
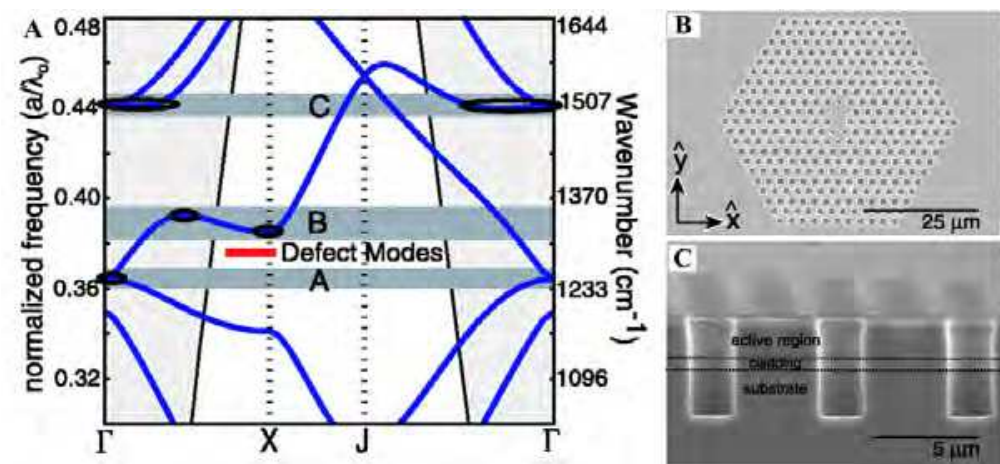

Fig. 2. (A) Theoretical simulation of $2 \mathrm{D}$ in-plane transverse magnetic (TM) photonic band structure. The flat-band region (A, B, and C) are indicated by dark grey horizontal bands. The red thick line indicates the highly localized defect modes. (B) Top-view scanning electron microscope (SEM) image of an etched device with a central defect. (C) Cross-section SEM of a portion of a photonic crystal QC device. (Colombelli F. , et al., 2003) 
This work also characterized the threshold behavior of the device in figure 4 . The lightoutput versus current characteristic of the device (red line) shows that the device fabrication was successful. However, the current voltage (I-V) relationship (blue line) presents the somewhat soft turn-on effect in the figure. The authors believe that a poor current confinement resulting from the absence of a mesa etched around the device should be the major reason. (Colombelli F. , et al., 2003) In addition, the estimation of its current density at threshold is 9 to $10 \mathrm{kA} / \mathrm{cm}^{2}$ which is higher than the conventional edge-emitting QCL.

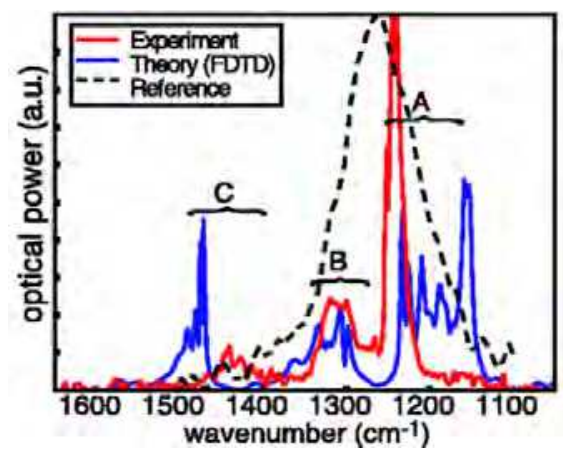

Fig. 3. Electroluminescence and FDTD-simulated spectrum for a device with a $=2.92 \mu \mathrm{m}$ and $r / a=0.30$. Dashed line indicates the reference spectrum collected from non-resonance area of the same device. (Colombelli F. , et al., 2003)

Nevertheless, the electrically pumped demonstration of surface-emitting photonic crystal QCL is an important step in the development of the practical photonic crystal microcavity lasers. It opens up the new horizons in device design and application in the mid-IR ranges using the combination of electronic and photonic bandgap engineering in semiconductor material. But this proof-of-concept demonstration also leaves some significant engineering issues, such as the high laser threshold $\left(9-10 \mathrm{kA} / \mathrm{cm}^{2}\right)$, and extremely low operation temperature $(10 \mathrm{~K})$, for further research exploration. (Bahriz, Moreau, \& Colombelli, 2007; Xu, et al., 2010)

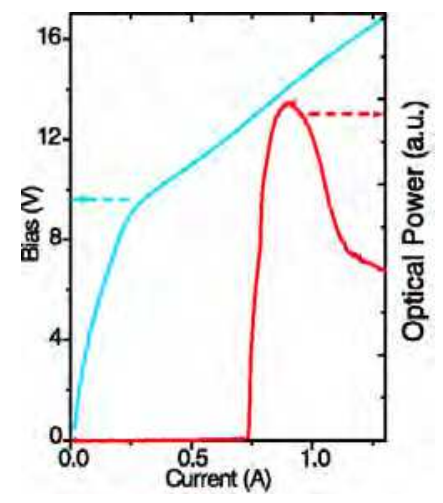

Fig. 4. Voltage versus current (V-I) and light-output versus injected current characteristics of a typical device at $10 \mathrm{~K}$, the peak power with a central defect is from $400 \mu \mathrm{W}$ to $1 \mathrm{~mW}$, while the ones without defect have less power output. (Colombelli F. , et al., 2003) 


\subsection{Surface-emitting photonic crystal distributed-feedback (DFB) laser based on III-V type-II "W" active regions}

In contrast to the mid-IR photonic crystal QCL lasers discussed previously, reported type-II "W" shape III-V surface-emitting photonic crystal DFB laser has a relatively shallow grating photonic crystal structure etched into the laser waveguide, as shown in figure 5A. In 2003, I. Vurgaftman and J. R. Meyer used a theory based on a time-domain Fourier-Galerkin (TDFG) numerical solution for simulating the surface-emitting photonic crystal DFB operation. They found that a "weak" 2D photonic crystal grating with only refractive-index modulation will yield high-efficiency surface emitting photonic crystal DFB emission into a second order single in-phase symmetric mode (Figure 5B), which is coherent over a wide device area. (Vurgaftman \& Meyer, 2003)
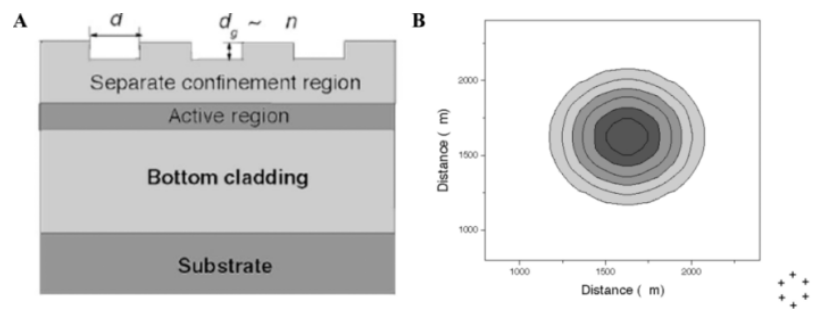

Fig. 5. (A) Cross section of surface emitting photonic crystal distributed feedback (DFB) laser; (B) simulated near-field profile for the single-lobed in-phase mode of hexagonal-lattice with transverse electric (TE) polarization. (Vurgaftman \& Meyer, 2003)

Following the theoretical studies guideline, the experimental demonstration of surface emitting photonic crystal DFB lasers with type-II "W" active regions emitting in the mid-IR range was reported by the same group in 2006. (Kim, et al., 2006) The epitaxial structure was grown on a (100) GaSb substrate using molecular beam epitaxy (MBE) method, consisting of a $3.5 \mu \mathrm{m}$ thick AlAsSb bottom optical cladding layer, an active region of 10 pairs of InAs/GaSb/AlSb quantum wells, and a $500 \mathrm{~nm}$ thick GaSb separate-confinement layer where the grating was applied. After growth, two triangular lattice grating patterns with etch depths of 27 and $60 \mathrm{~nm}$ were fabricated by using reactive ion etching and chemical wet etching, respectively. Figure 6 shows one titled view SEM image of a $60 \mathrm{~nm}$ deep photonic crystal DFB grating pattern processed after wet etching.

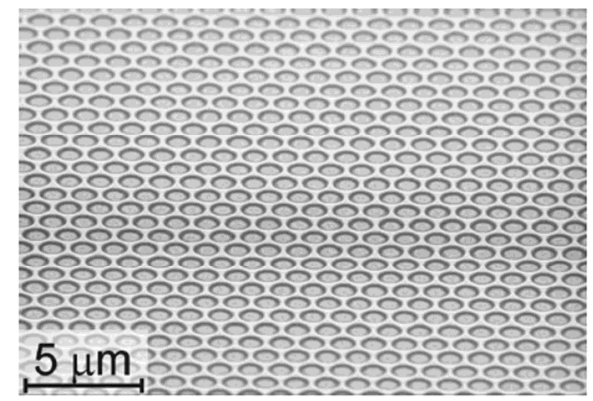

Fig. 6. Tilted view SEM image of the surface emitting photonic crystal DFB grating pattern with $60 \mathrm{~nm}$ etching depth. (Kim, et al., 2006) 
A high-resolution $(\sim 0.05 \mathrm{~nm})$ FTIR emission spectrum for the $27 \mathrm{~nm}$ deep surface emitting photonic crystal DFB lasers is demonstrated in Figure 7, with the $\mathrm{cw}$ pumping power $\sim 6 \mathrm{~W}$, and the pumping spot size $\sim 1.4 \mathrm{~mm}$. The emission peak with full width of half maximum (FWHM) $0.13 \mathrm{~nm}$ is observed. The confirmed side-mode suppression ratio of $>21 \mathrm{~dB}$ was limited by the apodization artifact in the instrument. (Kim, et al., 2006)

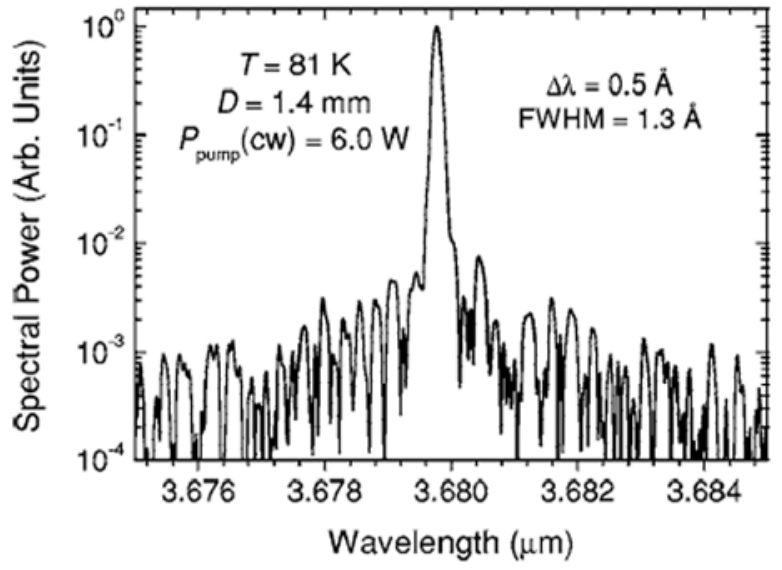

Fig. 7. FTIR spectrum for the surface emitting photonic crystal DFB laser with $27 \mathrm{~nm}$ etching depth at $81 \mathrm{~K}$. (Kim, et al., 2006)

Figure 8A shows the temperature dependent pulsed and $\mathrm{cw}$ light-light (L-L) characteristics for the photonic crystal DFB lasers with $27 \mathrm{~nm}$ etching depth. The slow increased threshold between $100 \mathrm{~K}$ and $140 \mathrm{~K}$ in contrast to the faster variation from $140 \mathrm{~K}$ to $180 \mathrm{~K}$, is due to the peak match between material gain and grating resonance, as presented in figure $8 \mathrm{~B}$. As indicated in Fig. 8A, the highest working temperature of the measured sample is $180 \mathrm{~K}$ under pulse mode and $81 \mathrm{~K}$ under $\mathrm{cw}$ mode. (Kim, et al., 2006) Although the authors claim that $180 \mathrm{~K}$ is below the maximum lasing temperature, there is no higher temperature value indicated in that letter. The maximum quantum efficiency calculated is only $0.6 \%$. They point out that these weak efficiencies are caused by the low pumping energy absorption in the active region. (Kim, et al., 2006) Besides on this reason, we believe that the weak lateral optical confinement due to the shallow etching grating could somehow enhance the optical leakages in the device. Therefore, the energy conversion rate would be affected.

Although the simulation theory for the surface emitting photonic crystal DFB lasers described above is totally different from the one discussed in the surface emitting photonic crystal QCL section, the physical mechanisms of these two devices are basically quite the same. Both of them use the coupled lasing modes resulting from the $2 \mathrm{D}$ photonic crystal distributed feedback mechanism. Therefore, these lasers have the unique coherent lasing mechanism over the wide 2D photonic crystal areas. However, due to the wide area cavity, the threshold current or optical pumping power would be relatively large. For a practical application, this issue should be improved. 
A

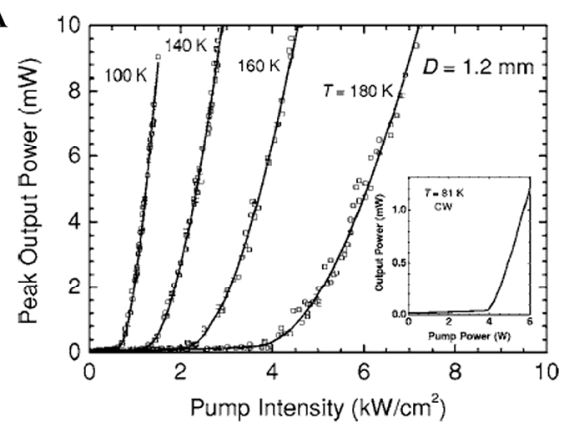

B

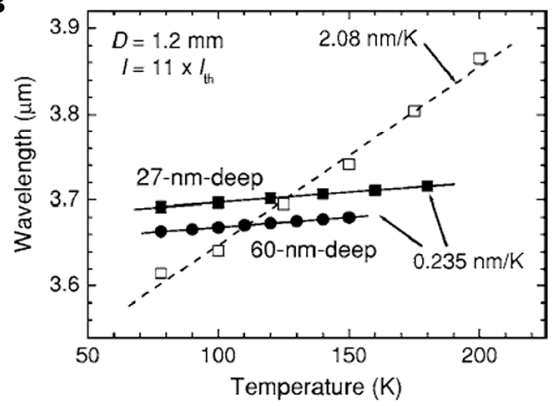

Fig. 8. (A) Temperature dependent pulsed light-light curves for the surface emitting photonic crystal DFB laser with $27 \mathrm{~nm}$ etching depth. Inset is the $\mathrm{cw}$ light-light curve at 81 K. (B) Peak emission position variation under the different temperature for the regular FP laser (open square), photonic crystal DFB lasers with $27 \mathrm{~nm}$ etching depth (solid square), and $60 \mathrm{~nm}$ etching depth (solid circle). (Kim, et al., 2006)

\subsection{Lead-salt based surface-emitting photonic crystal laser on $\mathrm{Si}$}

Lead salt or IV-VI semiconductor system has been used as a major material source for midIR diode lasers for a long time, because of its energy bandgap covering the wavelength region from 3 to $30 \mu \mathrm{m}$ (Preier, 1990; Ishida \& Fujiasyu, 2003; Tacke M. , 2000) and also its near two orders of magnitude lower non-radiative Auger recombination rates as compared to other conventional narrow band gap semiconductors (Findlay, et al., 1998). In this section, the realization of surface emitting photonic crystal lasers based on the lead salt compounds is described.

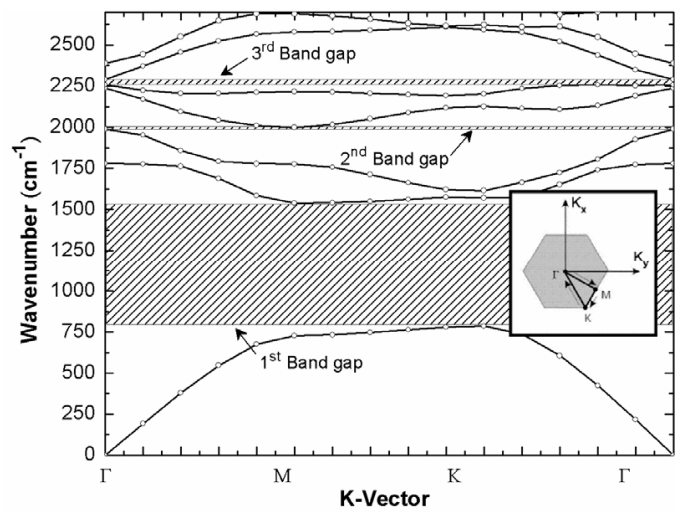

Fig. 9. Dispersion diagram of 2D transverse magnetic (TM) modes of the hexagonal photonic crystal holes array; the first Brillouin zone in reciprocal space is displayed in the inset.

(Weng, Ma, Wei, Xu, Bi, \& Shi, Mid-infrared surface-emitting photonic crystal microcavity light emitter on silicon, 2010)

In order to achieve the strong in-plane optical feedback, and also avoid etching of epitaxial films which alleviates the problem of surface recombination, the $2 \mathrm{D}$ photonic crystal 
honeycomb structure was fabricated onto the silicon substrate prior to the following IV-VI epitaxial layers growth. Using this modified experimental proposal, B. Weng et al. reported a PbSe/PbSrSe multiple quantum wells (MQW) mid-IR photonic crystal coupled light emitter in 2010. (Weng, Ma, Wei, Xu, Bi, \& Shi, Mid-infrared surface-emitting photonic crystal microcavity light emitter on silicon, 2010) For the initial proof-of-concept demonstration, they intentionally increased the designed values of interhole spacing and holes radius to micrometer range as to allow high tolerance for possible errors in etching and lateral growth. Therefore, the second and third order photonic bandgaps are moved into the mid-IR spectral region of interest, as presented in figure 9.

In the experiment, Si substrate was patterned by a hexagonal photonic crystal array using electron-beam (E-Beam) lithography. Defect cavity was created in the center of the pattern. RIE system was used to fabricate the photonic crystal patterned substrate with $3 \mu \mathrm{m}$ etching depth. After that, the epitaxial layer consisted of a seven-pair PbSe/PbSrSe MQW active region and $1 \mu \mathrm{m}$ optical confinement $\mathrm{BaF}_{2}$ top layer. (Weng, Ma, Wei, $\mathrm{Xu}, \mathrm{Bi}$, \& Shi, Midinfrared surface-emitting photonic crystal microcavity light emitter on silicon, 2010) Figure 10 shows top view SEM images of both patterned Si substrate after RIE etching process and after lead salt epitaxial growth.

A

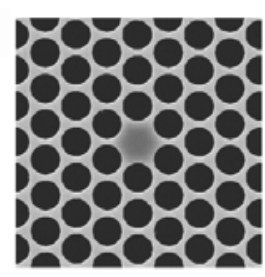

B

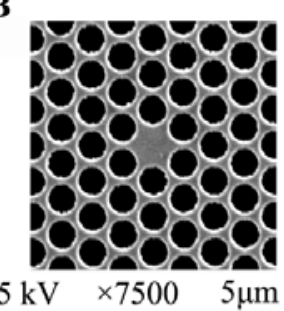

Fig. 10. Top view SEM images of (A) patterned Si(111) substrate; (B) PbSe/PbSrSe MQW structure grown on patterned $\mathrm{Si}(111)$. (Weng, Ma, Wei, Xu, Bi, \& Shi, Mid-infrared surfaceemitting photonic crystal microcavity light emitter on silicon, 2010)

The optical pumping emission spectra were characterized by a Bruker IFS 66/S FTIR spectrometer in step-scan mode. Measurements on the sample were taken at the areas both inside and outside the photonic crystal patterns. Detail setup information is discussed in the reference (Weng, Ma, Wei, Xu, Bi, \& Shi, Mid-infrared surface-emitting photonic crystal microcavity light emitter on silicon, 2010) Figure 11A presents the temperature dependent photoluminescence spectra of the un-patterned planar area on the sample. As it indicates, the emission peak shifting agrees well with the temperature dependent IV-VI semiconductor $Q W$ gain spectra. However, in figure 3B, there are two temperature-independent emission peaks at around 1960 and $2300 \mathrm{~cm}^{-1}$. The emission spectra were taken from the epitaxial layer grown on the 2D photonic crystal patterned Si substrate. Comparing with the theoretical simulation, these two peak positions align well with the calculated $2^{\text {nd }}$ and $3^{\text {rd }}$ band gaps. As we can see, there is only one emission mode emerging in each spectrum at a given temperature. The $2^{\text {nd }}$ order photonic crystal coupled emission mode at $1960 \mathrm{~cm}^{-1}$ dominates the range from $77 \sim 160$ $\mathrm{K}$ which the $3^{\text {rd }}$ one emerges from $180 \sim 270 \mathrm{~K}$. This phenomenon is due to the shift of the IVVI MQW gain spectra as shown in figure 11A. (Weng, Ma, Wei, Xu, Bi, \& Shi, Mid-infrared surface-emitting photonic crystal microcavity light emitter on silicon, 2010) 

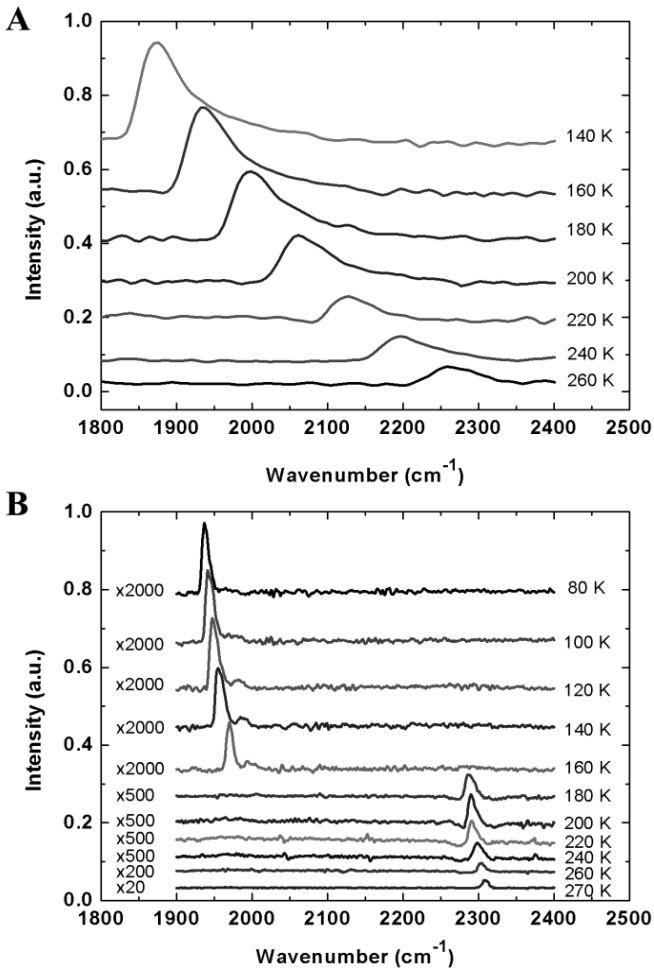

Fig. 11. Temperature-dependent light emission of PbSe/PbSrSe MQW (A) on un-patterned area and (B) patterned area on Si (111). (Weng, Ma, Wei, Xu, Bi, \& Shi, Mid-infrared surfaceemitting photonic crystal microcavity light emitter on silicon, 2010)

Meanwhile, the output emission power was calibrated by a standard blackbody reference source. The maximum emission peak-power is around $3.99 \mathrm{~W}$ at $100 \mathrm{~K}$, under $1 \mathrm{~kW}$ pumping peak-power excitation. Therefore, the quantum efficiency is $12.8 \%$. As can be seen from figure 12, the collected power from photonic patterned area is over two orders of magnitudes higher than that from planar area. The authors contribute such significant enhancement to several factors: (Weng, Ma, Wei, Xu, Bi, \& Shi, Mid-infrared surfaceemitting photonic crystal microcavity light emitter on silicon, 2010) First of all, the 2D photonic crystal structure forces all the photons generated to funnel through its given modes and be extracted, where the extraction efficiency of PL emission from the planar epilayer is low under the total reflection limitation. Secondly, the narrow beam divergence of the emission also increases the collected efficiency. Thirdly, great improvement in material quality due to the growth on patterned substrate could significantly reduce the material losses. (Weng, Zhao, Ma, Yu, Xu, \& Shi, 2010)

After the initial demonstration of their surface emitting 2D photonic crystal lead-salt light emitter, a new room temperature mid-IR surface emitting 2D photonic crystal laser on $\mathrm{Si}$ was successfully realized by B. Weng et al., (Weng, et al., Room temperature mid-infrared surface-emitting photonic crystal laser on silicon, 2011) recently. Up to date, this is the first mid-IR surface emitting photonic crystal semiconductor laser realized at room temperature. 


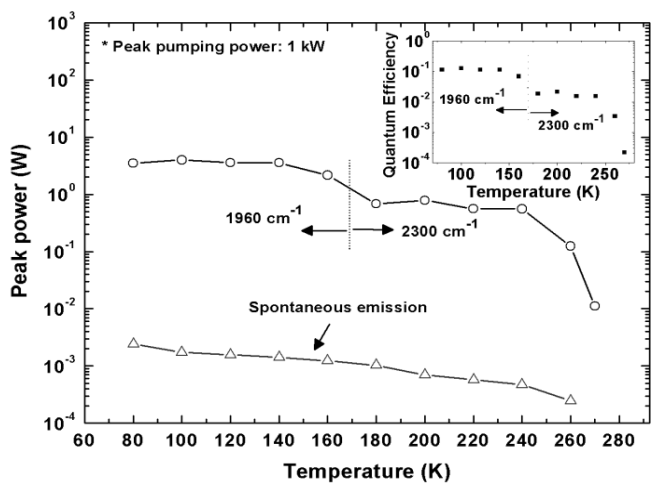

Fig. 12. Calibrated temperature dependent output emission power from $\mathrm{PbSe} / \mathrm{PbSrSe} \mathrm{MQW}$ structure on patterned and un-patterned areas. Quantum efficiency of the emission is show in the inset. (Weng, Ma, Wei, Xu, Bi, \& Shi, Mid-infrared surface-emitting photonic crystal microcavity light emitter on silicon, 2010)

As described previously in this section, for the easy proof-of-concept demonstration, the bandgaps for mode emissions aligning with the gain peak of a PbSe/PbSrSe QW were at cryogenic temperatures. Therefore, in order to obtain room temperature photonic crystal modulated mode emission, a modified photonic crystal dispersion diagram was designed which matches the room temperature gain peak of a designed PbSe/PbSrSe QW, presented in figure 13.

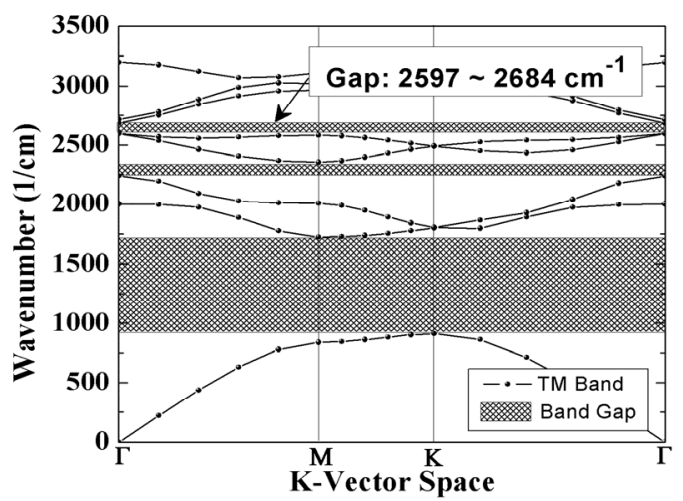

Fig. 13. Dispersion diagram of 2D TM modes of the designed photonic crystal array. (Weng, et al., Room temperature mid-infrared surface-emitting photonic crystal laser on silicon, 2011)

The photonic crystal structure fabrication on Si substrate and also the epitaxial layer growth procedures by MBE system both followed the methods reported in reference (Weng, et al., Room temperature mid-infrared surface-emitting photonic crystal laser on silicon, 2011). Figure 14A shows a top-view SEM image of patterned area with the defect on Si substrate after MBE growth. Later on, without any further processing, the emission spectra of the as grown sample were measured by the same Bruker FTIR system, and the signal was detected by a liquid $\mathrm{N}_{2}$ cooled InSb detector (Judson J-10D). Within the temperature range of $270 \sim 310 \mathrm{~K}$, 
there is only one temperature-independent emission peak observed. However, under the same pumping power, the emission spectrum at $310 \mathrm{~K}$ is below the threshold and exhibits a much broader emission linewidth. The phenomenon of the fixed peak position under different temperatures is similar to the reported result as shown in figure 11B. (Weng, $\mathrm{Ma}, \mathrm{Wei}, \mathrm{Xu}, \mathrm{Bi}$, \& Shi, Mid-infrared surface-emitting photonic crystal microcavity light emitter on silicon, 2010)
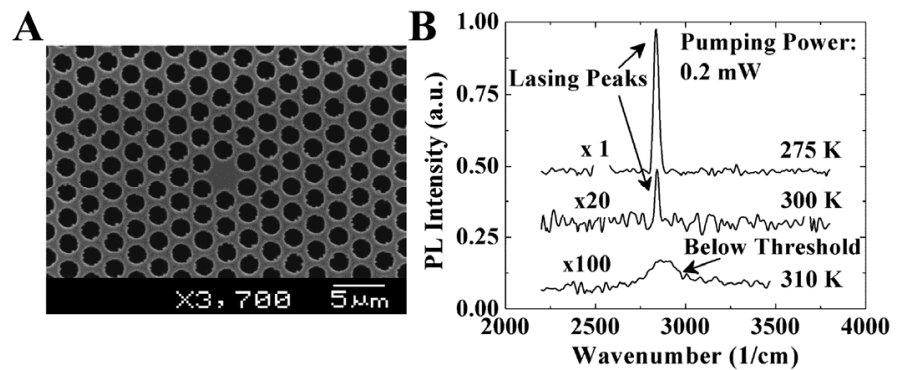

Fig. 14. (A) Top-view SEM image of PbSe/PbSrSe MQWs structure grown on patterned $\mathrm{Si}(111)$; (B) Temperature dependent light emission of the same sample. (Weng, et al., Room temperature mid-infrared surface-emitting photonic crystal laser on silicon, 2011)

Figure 15 shows the surface emitting 2D photonic crystal light emission spectrum at $300 \mathrm{~K}$ above threshold. As figure 15A indicates, the lasing peak doesn't perfectly match the designed emission wavelength $(\sim 3.85 \mu \mathrm{m})$ of the QW gain peak position on un-patterned area of the same sample. Comparing with the designed photonic bandgap matching with $3.85 \mu \mathrm{m}$, the after-processed sample has the lasing peak blue shifted to $3.52 \mu \mathrm{m}$. After checked with the dimension the etched pattern, it is found that, the after-etched radius is larger than the designed $1.1 \mu \mathrm{m}$, which is $1.14 \mu \mathrm{m}$. Simulation using the actual radius parameter shows a higher photonic bandgap which matches the experimental result. As indicated in figure 15B, lasing emission from the photonic crystal structure has multi-mode characteristics. Although the individual peaks could not totally be resolved experimentally due to the restriction by the instrument limitation, using Gaussian fitting function, there are four stimulated peaks resolved mathematically from the spectrum obtained by FTIR spectrometer. Their full width of half maximum (FWHM) values vary from $2.45 \mathrm{~nm}$ to 6.02 $\mathrm{nm}$, as presented. According to their design, these peaks are the photonic crystal coupled microcavity modes generated by the defect structure in the center of the array shown in figure 14A. (Weng, et al., Room temperature mid-infrared surface-emitting photonic crystal laser on silicon, 2011) Meanwhile, the authors point out some other mechanisms might contributing the multi-mode emissions, (Weng, et al., Room temperature mid-infrared surface-emitting photonic crystal laser on silicon, 2011) such as degeneracy splitting of dipole mode due to the structure imperfection (Painter, Vuckovic, \& Scherer, Defect modes of a two-dimensional photonic crystal in an optically thin dielectric slab, 1999), photonic band-edge mode emission (Colombelli R. , et al., 2004), etc.

The optical output power versus input pumping power density curve of the photonic crystal surface emitting laser was characterized and displayed in figure 16. A clear threshold behavior can be observed. As it indicates, the threshold pumping power density is approximately 24 $\mathrm{kW} / \mathrm{cm}^{2}$ at $300 \mathrm{~K}$. The relatively low threshold is mainly due to the reasons: (a). IV-VI epitaxial layer grown on holes-patterned $\mathrm{Si}$ substrate has better material quality than planar grown structure, therefore crystal defects and dislocations have less impact on the photonic crystal 
device which ultimately lowered non-radiative recombination rate; (b). with the help of 2D photonic crystal structure, group-velocity anomaly phenomenon could also have played an important role in a large reduction of the threshold.
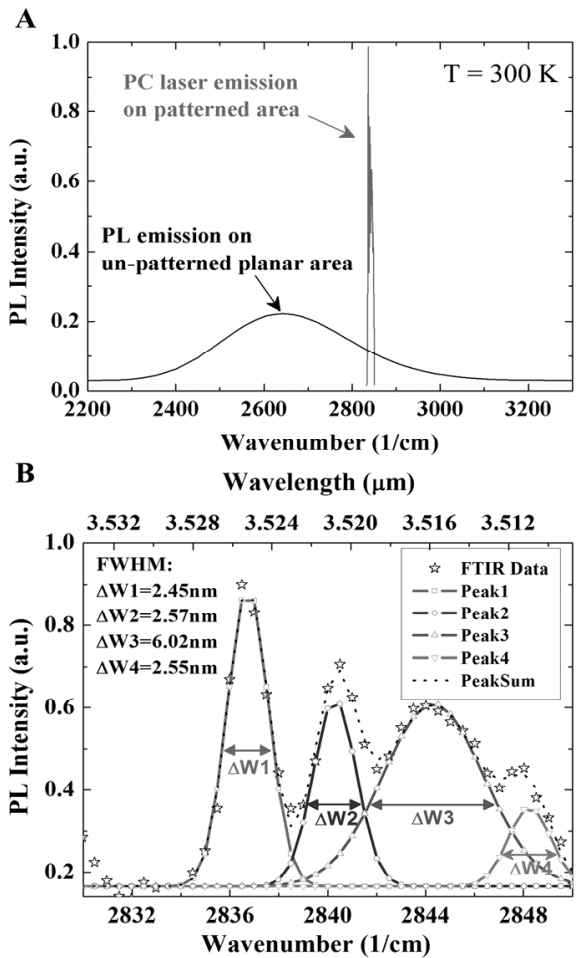

Fig. 15. (A) Comparison of photonic crystal emission spectrum above threshold and the light emission spectrum on un-patterned planar area at room temperature; (B) Multi-mode laser spectrum and linewidths determined by Gaussian function fitting. (Weng, et al., Room temperature mid-infrared surface-emitting photonic crystal laser on silicon, 2011)

As shown in figure 15, the peak emission powers were calibrated using a standard blackbody source. For the output power of $2.74 \mathrm{~mW}$, the quantum efficiency calculated is about $1 \%$. (Weng, et al., Room temperature mid-infrared surface-emitting photonic crystal laser on silicon, 2011) This low efficiency is mainly caused by the peak positions mismatch between photonic crystal coupled mode and the QW optical gain, as indicated in figure 15A. It is also pointed out that with the design modification to realign photonic crystal coupled mode and QW gain peak spectrum, once the peaks match up, significant efficiency increase would be realized.

\section{Conclusions}

In conclusion, surface emitting 2D photonic crystal lasers offer attractive properties as the midIR solid state laser sources. In particular, this group of lasers features intrinsic circular beam emission with small beam divergence, low threshold, single mode operation etc. Since the first 


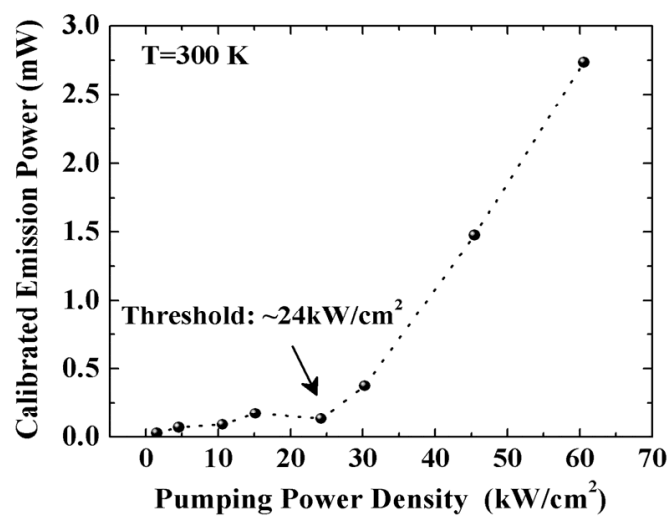

Fig. 16. Calibrated surface emitting photonic crystal laser peak output power as a function of the peak optical pumping power density at room temperature. (Weng, et al., Room temperature mid-infrared surface-emitting photonic crystal laser on silicon, 2011)

demonstration of the surface emitting 2D photonic crystal laser at $10 \mathrm{~K}$ in mid-IR range of electromagnetic field using intersubband cascade structure based on III-V material system, several attempts have been made in order to bring this type of devices up to the practical application level in the real world. Up to now, room temperature operation of the surface emitting 2D photonic crystal laser is only achieved by using IV-VI lead salt material system under pulsed optical excitation. But with further technological design and fabrication efforts, room temperature electrically pumped $\mathrm{CW}$ lasers should be feasible in near future, and this will definitely explore many promising applications in a wide variety of fields.

\section{References}

Altug, H., Englund, D., \& Vuckovic, J. (2006). Ultrafast photonic crystal nanocavity laser . Nature Physics, 2, 484.

Bahriz, M., Moreau, V., \& Colombelli, R. (2007). Design of mid-IR and Thz quantum cascade laser cavities with complete TM photonic bandgap. Optics Express, 15, 5948.

Bauer, A., Roßner, K., Lehnhardt, T., Kamp, M., Hofling, S., Worschech, L., et al. (2011). Midinfrared semiconductor heterostructure lasers for gas sensing applications. semiconductor science and technology, 26, 014032.

Colombelli, F., Srinivasan, K., Troccoli, M., Painter, O., Gmachl, C. F., Tennant, D. M., et al. (2003). Quantum cascade surface-emitting photonic crystal laser. Science, 302, 1374.

Colombelli, R., Srinivasan, K., Troccoli, M., Painter, O., Gmachl, C. F., Tennant, D. M., et al. (2004). Quantum cascade photonic-crystal microlasers. Proceedings of SPIE, 5365, 228.

Diehl, L., Bour, D., Corzine, S., Zhu, J., Hfler, G., Loncar, M., et al. (2006). High-power quantum cascade lasers grown by low-pressure metal organic vapor-phase epitaxy operating in continuous wave above 400K. Applied Physics Letters, 88, 201115.

Findlay, P. C., Pidgon, C. R., Kotitschke, R., Hollingworth, A., Murdin, B. N., Langerak, C. J., et al. (1998). Auger recombination dynamics of lead salts under picosecond freeelectron-laser excitation. Physical Review B, 58, 12908.

H.C. Casey, J., \& Panish, M. B. (1978). Heterostructure Lasers. San Diego, USA.

Ishida, A., \& Fujiasyu, H. (2003). Lead Chalcogenides: Physics and Applications. (Khokhlov, Ed.) New York, USA: Taylor and Francis. 
Kim, M., Kim, C. S., Bewley, W. W., Lindle, J. R., Canedy, C. L., Vurgaftman, I., et al. (2006). Surface-emitting photonic-crystal distributed-feedback laser for the midinfrared. Applied Physics Letters, 88, 191105.

Loncar, M., Yoshie, T., Scherer, A., Gogna, P., \& Qiu, Y. (2002). Low-threshold photonic crystal laser. Applied Physics Letters, 81, 2680.

Nojima, S. (1998). Enhancement of optical gain in two-dimensional photonic crystals with active lattice points. Jpn. J. Appl. Phys., 37, 565.

Painter, O., Lee, R. K., Scherer, A., Yariv, A., O'Brien, J. D., Dapkus, P. D., et al. (1999). Twodimensional photonic band-gap defect mode laser. Science, 284, 1819.

Painter, O., Vuckovic, J., \& Scherer, A. (1999). Defect modes of a two-dimensional photonic crystal in an optically thin dielectric slab. Journal of the Optical Society of America B , 16, 275.

Park, H., Hwang, J., Huh, J., Ryu, H., \& Lee, Y. (2001). Nondegenerate monopole-mode twodimensional photonic band gap laser. Applied Physics Letters, 79, 3032.

Preier, H. (1990). Physics and applications of IV-VI compound semiconductor lasers . Semiconductor Science and Technology, 5, S12.

Slivken, S., Evans, A., Zhang, W., \& Razeghi, M. (2007). High-power, continuous-operation intersubband laser for wavelengths greater than $10 \mu \mathrm{m}$. Applied Physics Letters, 90, 151115.

Springholz, G., Schwarzl, T., \& Heiss, W. (2006). Mid-infrared Vertical Cavity Surface Emitting Lasers based on the Lead Salt Compounds. Mid-infrared Semiconductor Optoelectronics, Springer Series in Optical Sciences, 118, 265.

Tacke, M. (2000). Long Wavelength Infrared Emitters Based on Quantum Wells and Superlattices. (M. Helm, Ed.) Amsterdam, Holand: Gordon and Breach Science Publishers.

Tacke, M. (2001). Lead-salt lasers. Phil. Trans. R. Soc. Lond. A, 359, 547.

Tittel, F. K., Richter, D., \& Fried, A. (2003). Solid State Mid-Infrared Lasers Sources. (S. I. T, \& V. K. L, Eds.) Berlin: Springer-Verlag.

Vurgaftman, I., \& Meyer, J. R. (2003). Design optimization for high-brightness surface-emitting photonic-crystal distributed-feedback lasers. IEEE Journal of Quantum electronics, 39, 689.

Weng, B., Ma, J., Wei, L., Li, L., Qiu, J., Xu, J., et al. (2011). Room temperature mid-infrared surface-emitting photonic crystal laser on silicon. Applied Physics Letters, 99, 221110.

Weng, B., Ma, J., Wei, L., Xu, J., Bi, G., \& Shi, Z. (2010). Mid-infrared surface-emitting photonic crystal microcavity light emitter on silicon. Applied Physics Letters, 97, 231103.

Weng, B., Zhao, F., Ma, J., Yu, G., Xu, J., \& Shi, Z. (2010). Elimination of threading dislocations in as-grown PbSe film on patterned $\mathrm{Si}(111)$ substrate using molecular beam epitaxy. Applied Physics Letters, 96, 251911.

Xu, G., Colombelli, R., Braive, R., Beaudoin, G., Gratiet, L. L., Talneau, A., et al. (2010). Surface-emitting mid-infrared quantum cascade lasers with high-contrast photonic crystal resonators. Optics Express, 18, 11979.

Yang, R. Q. (1995). Infrared laser based on intersubbandtransitions in quantum wells. Superlattices and Microstructures, 17, 77.

Yang, R. Q., Bradshaw, J. L., Bruno, J. D., Pham, J. T., Wortman, D. E., \& Tober, R. L. (2002). Room temperature type-II interband cascade laser. Applied Physics Letters, 81, 397.

Yu, J. S., Darvish, S. R., Evans, A., Nguyen, J., Slivken, S., \& Razeghi, M. (2006). Roomtemperature continuous-wave operation of quantum-cascade lasers at $\lambda \sim 4 \mu \mathrm{m}$. Applied Physics Letters, 88, 041111.

Zhao, F., Wu, H., Majumdar, A., \& Shi, Z. (2003). Continuous wave optically pumped leadsalt mid-infrared quantum-well vertical-cavity surface-emitting lasers. Applied Physics Letters, 83, 5133. 


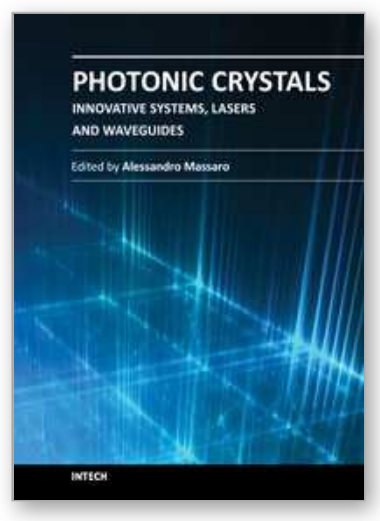

\author{
Photonic Crystals - Innovative Systems, Lasers and Waveguides \\ Edited by Dr. Alessandro Massaro
}

ISBN 978-953-51-0416-2

Hard cover, 348 pages

Publisher InTech

Published online 30, March, 2012

Published in print edition March, 2012

The second volume of the book concerns the characterization approach of photonic crystals, photonic crystal lasers, photonic crystal waveguides and plasmonics including the introduction of innovative systems and materials. Photonic crystal materials promises to enable all-optical computer circuits and could also be used to make ultra low-power light sources. Researchers have studied lasers from microscopic cavities in photonic crystals that act as reflectors to intensify the collisions between photons and atoms that lead to lazing, but these lasers have been optically-pumped, meaning they are driven by other lasers. Moreover, the physical principles behind the phenomenon of slow light in photonic crystal waveguides, as well as their practical limitations, are discussed. This includes the nature of slow light propagation, its bandwidth limitation, coupling of modes and particular kind terminating photonic crystals with metal surfaces allowing to propagate in surface plasmon-polariton waves. The goal of the second volume is to provide an overview about the listed issues.

\title{
How to reference
}

In order to correctly reference this scholarly work, feel free to copy and paste the following:

Binbin Weng and Zhisheng Shi (2012). Mid-Infrared Surface-Emitting Two Dimensional Photonic Crystal Semiconductor Lasers, Photonic Crystals - Innovative Systems, Lasers and Waveguides, Dr. Alessandro Massaro (Ed.), ISBN: 978-953-51-0416-2, InTech, Available from: http://www.intechopen.com/books/photoniccrystals-innovative-systems-lasers-and-waveguides/mid-infrared-surface-emitting-two-dimensional-photoniccrystal-semiconductor-lasers

\section{INTECH}

open science | open minds

\section{InTech Europe}

University Campus STeP Ri

Slavka Krautzeka 83/A

51000 Rijeka, Croatia

Phone: +385 (51) 770447

Fax: +385 (51) 686166

www.intechopen.com

\section{InTech China}

Unit 405, Office Block, Hotel Equatorial Shanghai

No.65, Yan An Road (West), Shanghai, 200040, China

中国上海市延安西路65号上海国际贵都大饭店办公楼405单元

Phone: +86-21-62489820

Fax: $+86-21-62489821$ 
(C) 2012 The Author(s). Licensee IntechOpen. This is an open access article distributed under the terms of the Creative Commons Attribution 3.0 License, which permits unrestricted use, distribution, and reproduction in any medium, provided the original work is properly cited. 